\title{
MBP-FGF2-Immobilized Matrix Maintains Self-Renewal and Myogenic Differentiation Potential of Skeletal Muscle Stem Cells
}

\author{
Jay Prakash Sah ${ }^{1,2, *}$, Nguyen Thi Thu Hao ${ }^{1,2, *}$, Yunhye Kim ${ }^{1,2}$, Tamar Eigler $^{3}$, Eldad Tzahor ${ }^{3}$, \\ Sang-Heon $\mathrm{Kim}^{4}$, Yongsung Hwang ${ }^{1,2}$, Jeong Kyo Yoon ${ }^{1,2}$ \\ ${ }^{I}$ Soonchunhyang Institute of Medi-bio Science, Soon Chun Hyang University, Cheonan, Korea \\ ${ }^{2}$ Department of Integrated Biomedical Science, Graduate School, Soon Chun Hyang University, Asan, Korea \\ ${ }^{3}$ Department of Molecular Cell Biology, Weizmann Institute of Science, Rehovot, Israel \\ ${ }^{4}$ Center for Biomaterials, Biomedical Research Institute, Korea Institute of Science and Technology, Seoul, Korea
}

\begin{abstract}
The robust capacity of skeletal muscle stem cells (SkMSCs, or satellite cells) to regenerate into new muscles in vivo has offered promising therapeutic options for the treatment of degenerative muscle diseases. However, the practical use of SkMSCs to treat muscle diseases is limited, owing to their inability to expand in vitro under defined cultivation conditions without loss of engraftment efficiency. To develop an optimal cultivation condition for SkMSCs, we investigated the behavior of SkMSCs on synthetic maltose-binding protein (MBP)-fibroblast growth factor 2 (FGF2)-immobilized matrix in vitro. We found that the chemically well-defined, xeno-free MBP-FGF2-immobilized matrix effectively supports SkMSC growth without reducing their differentiation potential in vitro. Our data highlights the possible application of the MBP-FGF2 matrix for SkMSC expansion in vitro.
\end{abstract}

Keywords: Skeletal muscle stem cell, Satellite cell, MBP-FGF2, Self-renewal, Myogenic differentiation

\section{Introduction}

Skeletal muscle stem cells (SkMSCs), called as satellite cells, are the undifferentiated cells residing between the basal lamina and the plasma membrane of myofibers. These cells play significant roles in the regeneration of the damaged muscle or maintenance of skeletal muscle ho- meostasis $(1,2)$. Under normal physiological conditions, SkMSCs remain in a quiescent state; however, these cells undergo vigorous proliferation in response to muscle injury or under degenerative pathological condition through the stimulation of multiple mitogenic factors and differentiate into myocytes, which may fuse to the pre-existing myofibers or form nascent myofibers $(3,4)$.

\footnotetext{
Received: December 6, 2018, Revised: December 19, 2018, Accepted: December 20, 2018, Published online: February 28, 2019 Correspondence to Jeong Kyo Yoon

Soonchunhyang Institute of Medi-bio Science (SIMS), Soon Chun Hyang University, 25 Bongjeong-ro, Dongnam-gu, Cheonan 31151, Korea Tel: +82-41-413-5016, Fax: +82-41-413-5006, E-mail: jkyoon@sch.ac.kr

Co-Correspondence to Yongsung Hwang

Soonchunhyang Institute of Medi-bio Science (SIMS), Soon Chun Hyang University, 25 Bongjeong-ro, Dongnam-gu, Cheonan 31151, Korea Tel: +82-41-413-5017, Fax: +82-41-413-5006, E-mail: yshwang0428@sch.ac.kr

Co-Correspondence to Sang-Heon Kim

Center for Biomaterials, Korea Institute of Science and Technology, Hwarang-ro 14-gil, Seongbook-gu, Seoul 02792, Korea

Tel: +82-2-958-5344, Fax: +82-2-958-5308, E-mail: skimbrc@kist.re.kr

*These authors contributed equally to this study.

(c) This is an open-access article distributed under the terms of the Creative Commons Attribution Non-Commercial License (http://creativecommons.org/licenses/by-nc/4.0/), which permits unrestricted non-commercial use, distribution, and reproduction in any medium, provided the original work is properly cited.

Copyright (c) 2019 by the Korean Society for Stem Cell Research
} 
The robust proliferative capacity and myogenic differentiation potential of SkMSCs have gathered interest among researchers to develop cell-based therapeutics for the treatment of degenerative muscle diseases (5). Despite significant progress in the understanding of the major signaling mechanisms to control cell proliferation and differentiation, SkMSCs show limited in vivo engraftment efficiency upon in vitro expansion (6). Furthermore, in vitro culture condition to promote initial adhesion and growth of SkMSCs require the use of Matrigel, a chemically undefined and animal-derived mixtures of extracellular matrix (7). These limitations act as a major hurdle in the development of SkMSC-based therapeutics. While several studies have shown that the manipulation of key myogenic transcription factors or signaling pathways may improve the engraftment efficiency of SkMSCs, other evidences indicate that the extracellular in vitro niche may not completely recapitulate the in vivo environment $(8,9)$. A biomaterial-based synthetic substrate with skeletal muscle-matching elasticity was shown to effectively support the in vitro self-renewal of SkMSCs and their subsequent in vivo engraftment within host tissues (10). Hence, the development of a well-defined synthetic niche to culture SkMSCs in vitro without any loss of their phenotypes is critically important to enhance therapeutic use (11).

We have previously developed a novel method to immobilize fibroblast growth factor 2 (FGF2) into tissue culture polystyrene substrates (12) and demonstrated that the FGF2-immobilized matrix controls the stem cell lineage commitment into targeted cell types through heparan sulfate proteoglycan (HSPG)-mediated cell adhesion behavior and the consequent cell-matrix interactions $(13,14)$. In this study, we extended the application of the maltose-binding protein (MBP)-FGF2 matrix to SkMSC culture. Our results demonstrate that the MBP-FGF2-immobilized matrix can support initial adhesion and activation of freshly isolated quiescent SkMSCs. In addition, the SkMSCs cultured on the MBP-FGF2 matrix can proliferate and subsequently differentiate into myotubes, similar to the SkMSCs cultured on Matrigel. Thus, our chemically well-defined and xeno-free cell culture platform provides a novel method to expand SkMSCs and may accelerate the potential use of SkMSCs for therapeutic purposes.

\section{Materials and Methods}

\section{Preparation of MBP-FGF2-immobilized cell culture substrates}

MBP was introduced to immobilize FGF2 into the hy- drophobic surface of tissue culture polystyrene $(12,14)$. MBP-FGF2 fusion protein was expressed and produced from Escherichia coli K12 TB1 carrying a pMAL-FGF2 plasmid that contains human FGF2 165 cDNA. The recombinant MBP-FGF2 was prepared in phosphate-buffered saline at varying concentrations $(10,25,30$, and 50 $\mu \mathrm{g} / \mathrm{mL}$ ) and applied to the surface of cell culture vessel following incubation for $1 \mathrm{~h}$ at room temperature $\left(24^{\circ} \mathrm{C}\right)$, followed by overnight incubation at $4^{\circ} \mathrm{C}$. To minimize non-specific cell adhesion, the surface of the cell culture vessel was blocked with $1 \%$ bovine serum albumin for 1 $\mathrm{h}$ at room temperature prior to cell seeding.

\section{Isolation and culture of SkMSCs}

All animal experimental procedures were performed according to the protocol (SCH16-0029) approved by Soonchunhyang University Animal Care and Use Committee. Quiescent SkMSCs (CD31-, CD45-, and Sca-1-negative and integrin $\alpha$ 7-positive) were isolated from hindlimb muscles of 6- to 8-week-old ICR mice by magneticactivated cell sorting as previously described (15). The isolated quiescent SkMSCs were immediately seeded and cultured in growth medium (Ham-F10, 20\% horse serum, 1\% penicillin-streptomycin, $5 \mathrm{ng} / \mathrm{mL}$ of human FGF2 (PeproTech, Rocky Hill, NJ, USA) in $5 \% \mathrm{CO}_{2}$ incubator at $37^{\circ} \mathrm{C}$.

SkMSCs at exponential growth phase were prepared from the gastrocnemius muscle of 6- to 8-week-old ICR mice as previously described (16). SkMSCs were plated on Matrigel-coated culture dishes and cultured until 70 80\% confluence in AmnioMAX-II complete medium (Thermo Fisher Scientific, Waltham, MA, USA). Cells were detached from culture plates with $0.05 \%$ trypsin and preplated for $10 \mathrm{~min}$ in an uncoated cell culture dish to remove fibroblasts before seeding for experiments. Myogenic differentiation was induced using Dulbecco's modified Eagle's medium (DMEM) supplemented with 2\% heat-inactivated horse serum.

\section{Cell proliferation assay}

SkMSCs were seeded at a density of $2 \times 10^{3}$ cells/well in 96-well plates coated with either Matrigel or MBPFGF2 and cultured in growth medium in the absence or presence of $5 \mathrm{ng} / \mathrm{mL}$ of human FGF2. Cell proliferation rate was measured using CellVia enhanced cell viability assay kit (Ab Frontier, Seoul, Korea). Briefly, $10 \mu \mathrm{L}$ of CellVia reagent was added to each well and the cells were incubated for $1 \mathrm{~h}$ at $37^{\circ} \mathrm{C}$ in $5 \% \mathrm{CO}_{2}$ atmosphere, and the absorbance of each well was measured at $450 \mathrm{~nm}$ using a Multi Scan GO spectrophotometer (Thermo Fisher Scientific, Finland). 


\section{Immunofluorescence staining and microscopy}

Immunofluorescence staining was performed as previously described (17). Primary antibodies against myogenin (1:100, sc-52903, Santa Cruz Biotechnology, Dallas, TX, USA), MYOD ( $1: 250$, sc-377460, Santa Cruz Biotechnology), myosin heavy chain (MyHC; $0.3 \mu \mathrm{g} / \mathrm{mL}$, MF20, Developmental Studies Hybridoma Bank, Iowa City, IA, USA), and vinculin (1:100, ab129002, Abcam, Cambridge, MA, USA) were used. Phalloidin-fluorescein isothiocyanate $(5 \mu \mathrm{g} / \mathrm{mL}$, P5282, Sigma, St. Louis, MO, USA) was used to detect F-actin. Matching secondary antibodies conjugated to Alexa Fluor 488 (1: 400, A21121, Thermo Fisher Scientific) or Cy3 (1:400, A10520, Thermo Fisher Scientific; $1: 500,115-165-207$, Jackson ImmunoResearch Laboratories, West Grove, PA, USA) were used. Cell nuclei were counter-stained with 4', 6-diamidino-2-phenylindole (DAPI, Invitrogen, Waltham, MA, USA). Cell images were obtained using Nikon digital SLR camera (DS-i2) attached to Nikon eclipse Ti-U inverted microscope.

For confocal microscopy, cells were grown in Lab-Tek II eight-well chamber slide (154534, Nunc, Waltham, MA, USA) coated with either Matrigel or MBP-FGF2. Subcellular localization of vinculin and F-actin was imaged using confocal laser scanning microscopy (Zeiss LSM 710 META, Jena, Germany).

\section{Statistical analysis}

All results are presented as the mean \pm standard error of mean (SEM) and were tested by comparing two experimental groups using the two-tailed Student's $t$-test. Graph and bar diagram were generated using GraphPad Prism software. $p$-values are indicated as $* p \leq 0.05, * * p \leq 0.01$, $* * * \mathrm{p} \leq 0.001$, and $\mathrm{p}$-values $\leq 0.05$ were considered statistically significant.

\section{Results and Discussion}

\section{MBP-FGF2-immobilized matrix can support the activation and proliferation of SkMSCs}

Previous studies have shown that the first step in the myogenic differentiation of quiescent SkMSCs is the activation of myogenic markers, as evident from the induction of $\mathrm{MyoD}$ expression. As a consequence, the activated SkMSCs rapidly proliferate until being committed to enter myogenic differentiation program (18). To investigate whether MBP-FGF2-immobilized matrix could support the initial adhesion and activation of quiescent SkMSCs in a concentration-dependent manner while inhibiting their spontaneous differentiation during in vitro ex- pansion, we examined the expression of $\mathrm{MyoD}$, an indicator of activated SkMSCs, and myogenin, a marker for the onset of myogenic differentiation, in SkMSCs cultured on Matrigel or MBP-FGF2 matrix. We seeded and cultured freshly isolated SkMSCs for up to $48 \mathrm{~h}$ and observed a slight decrease in the number of SkMSCs that adhered to the MBP-FGF2-immobilized matrix as compared to Matrigel (data not shown). As shown in Fig. 1, the majority (approximately 98\%) of the adhered SkMSCs cultured on the MBP-FGF2-immobilized matrix showed robust expression of $\mathrm{MyoD}$, consistent with the observation reported for the cells cultivated on Matrigel substrate. In contrast to MyoD expression pattern, myogenin expression level remained very low (less than $2 \%$ ) in the cells cultured on both Matrigel and the MBP-FGF2-immobilized matrix. These results suggest that SkMSCs grown on the MBP-FGF2-immobilized matrix efficiently undergo activation process and mostly remain in their undifferentiated states.

We examined the efficacy of the MBP-FGF2 immobilized matrix in supporting SkMSC proliferation. To determine whether the MBP-FGF2-immobilized matrix alone is sufficient to support the expansion of SkMSCs, SkMSCs were cultured on the MBP-FGF2-immobilized matrix at different concentrations in the absence of sFGF2 for 4 days. As shown in Fig. 2A, 2B, SkMSCs cultured on the MBP-FGF2 matrix showed a steady growth pattern in the absence of sFGF2 without any dose-dependent pattern; the proliferation rate of these cells was approximately $40 \sim 50 \%$ of that reported for the SkMSCs cultivated in the presence of sFGF2. In contrast, the cells cultured on BSA substrate completely failed to adhere and proliferate even in the presence of sFGF2. Therefore, the MBP-FGF2-immobilized matrix may exhibit decent mitogenic activity.

We evaluated the proliferative capacity of the SkMSCs cultured on the MBP-FGF2-immobilized matrix with varying concentrations in the presence of sFGF2. SkMSCs cultured on Matrigel-coated substrates in the presence of sFGF2 exhibited a robust proliferation profile during 4 days of culture (Fig. 2C). SkMSCs cultured on the MBPFGF2-immobilized matrix also showed strong proliferation ability in a dose-independent manner, but their proliferation was slightly slower than that observed for the cells cultured on Matrigel-coated substrate. Considering that lesser number of SkMSCs were adhered to the MBP-FGF2-immobilized matrix at the beginning of culture, our results suggest that SkMSCs, once attached to the matrix, may competently proliferate. Taken together, we conclude that the MBP-FGF2-immobilized matrix ef- 
A

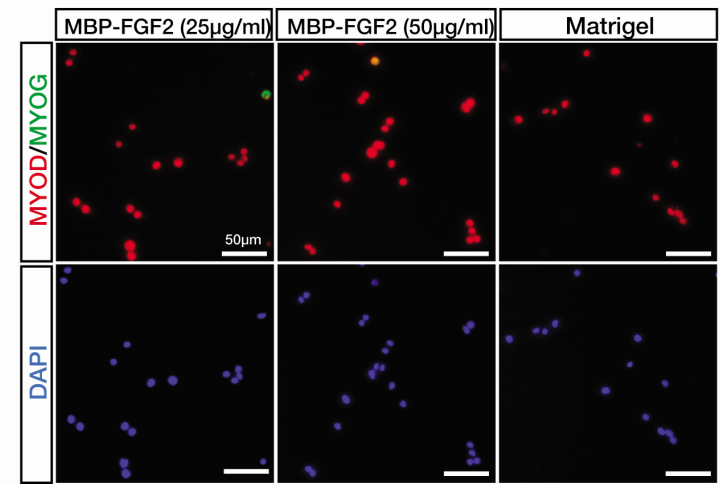

B

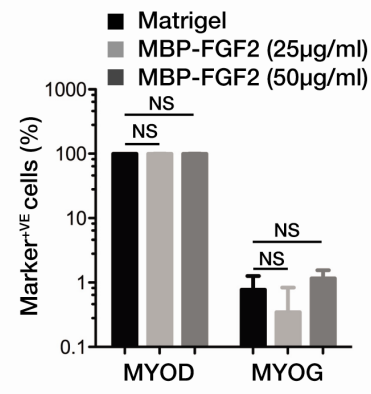

Fig. 1. MBP-FGF2-immobilized matrix can support the activation of SkMSCs. (A) Immunofluorescence staining images for the expression of myogenic markers, MyoD and myogenin (MYOG), in quiescent SkMSCs grown on Matrigel or MBP-FGF2 matrix for $48 \mathrm{~h}$ in growth medium. Cell nuclei were counterstained with DAPI. Scale bar, $50 \mu \mathrm{m}$. (B) Quantitation of MyoD- and MYOG-positive cells. Marker-positive cells are presented as the percentage of total cell number from triplicate samples. Error bars indicate standard error of the mean (SEM). NS, no statistical significance.

A

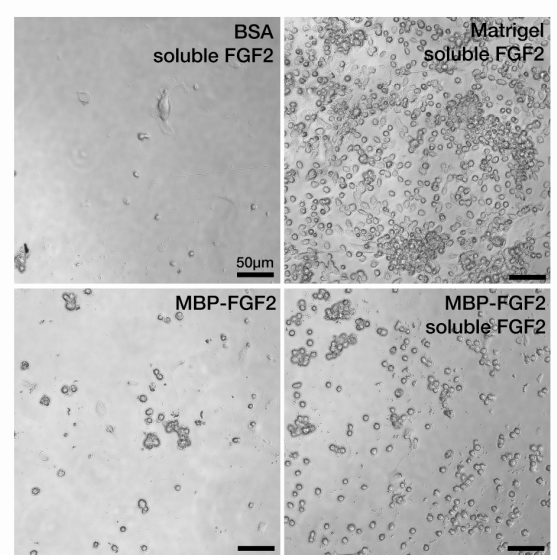

B

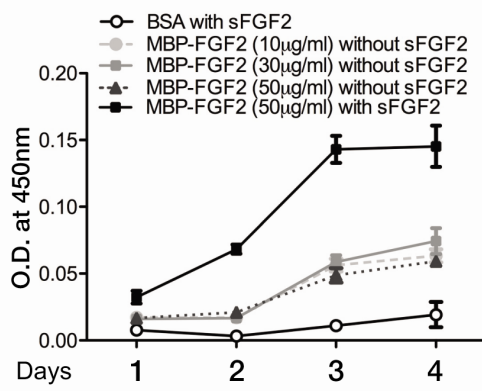

C

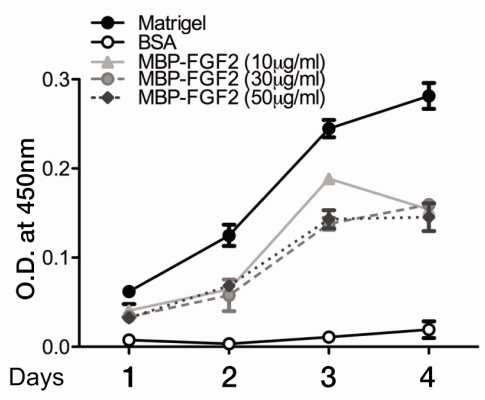

Fig. 2. SkMSCs competently proliferate on the MBP-FGF2-immobilized matrix. (A) Phase-contrast microscopic images of proliferating SkMSCs cultured on Matrigel or MBP-FGF2 matrix-coated surface with or without FGF2 supplementation in the growth medium for 4 days. Scale bar, $50 \mu \mathrm{m}$. (B, C) Determination of live SkMSC density with a modified MTS assay. The MTS assay was performed daily during the time course of 4 days. SkMSCs were cultured on BSA-, MBP-FGF2-, or Matrigel-coated 96-well plates in the absence (B) or presence (C) of soluble FGF2 (5 ng/mL) as indicated. Viable cell density was determined from the optical density (O.D.) at $450 \mathrm{~nm}$ wavelength. Experiments were performed in triplicates. Data represent normalized mean values with SEM.

fectively supports SkMSC proliferation and the addition of sFGF2 to the medium may synergistically enhance SkMSC proliferation.

\section{SkMSCs retain their myogenic potential on MBP-FGF2-immobilized matrix}

To determine whether SkMSCs cultured on the MBPFGF2-immobilized matrix maintain myogenic differentiation potential, we measured myogenic differentiation and cell fusion indices in SkMSCs expanded on MBPFGF2-immobilized matrix. Freshly isolated SkMSCs were expanded using both Matrigel and MBP-FGF2-immobi- lized matrix. The cells were reseeded on Matrigel-coated cell culture vessels in differentiation medium and immediately subjected to myogenic differentiation. After 3 days of differentiation, the cells were immunostained for sarcomeric MyHC expression.

As shown in Fig. 3A, SkMSCs precultured on the MBP-FGF2-immobilized matrix were able to differentiate into myotubes with an efficiency comparable to that of the cells precultured on Matrigel. Very similar differentiation indices were observed for both Matrigel- and MBP-FGF2 matrix-precultured cells (Fig. 3B). We noticed an interesting but not significant gradual increment in differ- 
A

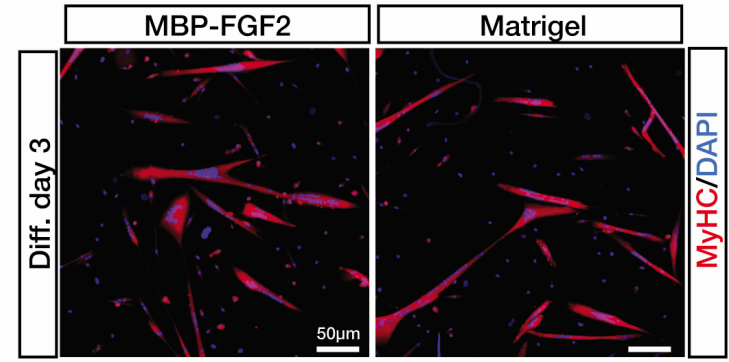

B

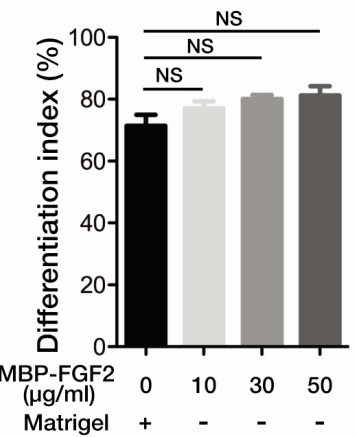

C

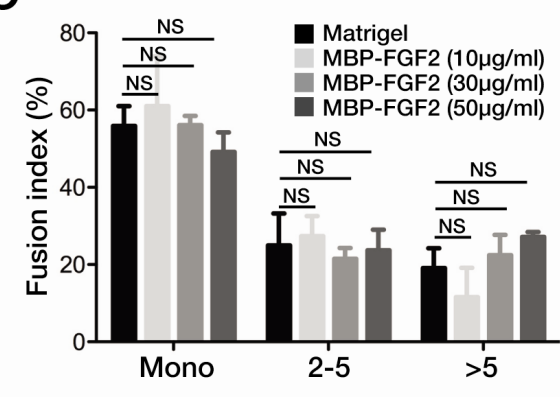

Fig. 3. SkMSCs maintain robust myogenic potential on MBP-FGF2mmobilized matrix. (A) Immunofluorescence staining images for $\mathrm{MyHC}$ expression in the SkMSCs cultured on Matrigel- or MBP-FGF2 (50 $\mu \mathrm{g} / \mathrm{mL})$ coated surfaces at differentiation day 3. Cell nuclei were counterstained with DAPI. Scale bar, $50 \mu \mathrm{m}$. (B) To calculate differentiation indices after 3 days, MyHC-positive cell nuclei were counted and expressed as the percentage of total cell nuclei. (C) Fusion indices were shown as a distribution of MyHC-positive cells based on cell nucleus number; mono, 2 5, and more than 5 nuclei. Experiments were performed in triplicates. Error bars indicate SEM. NS, no statistical significance.
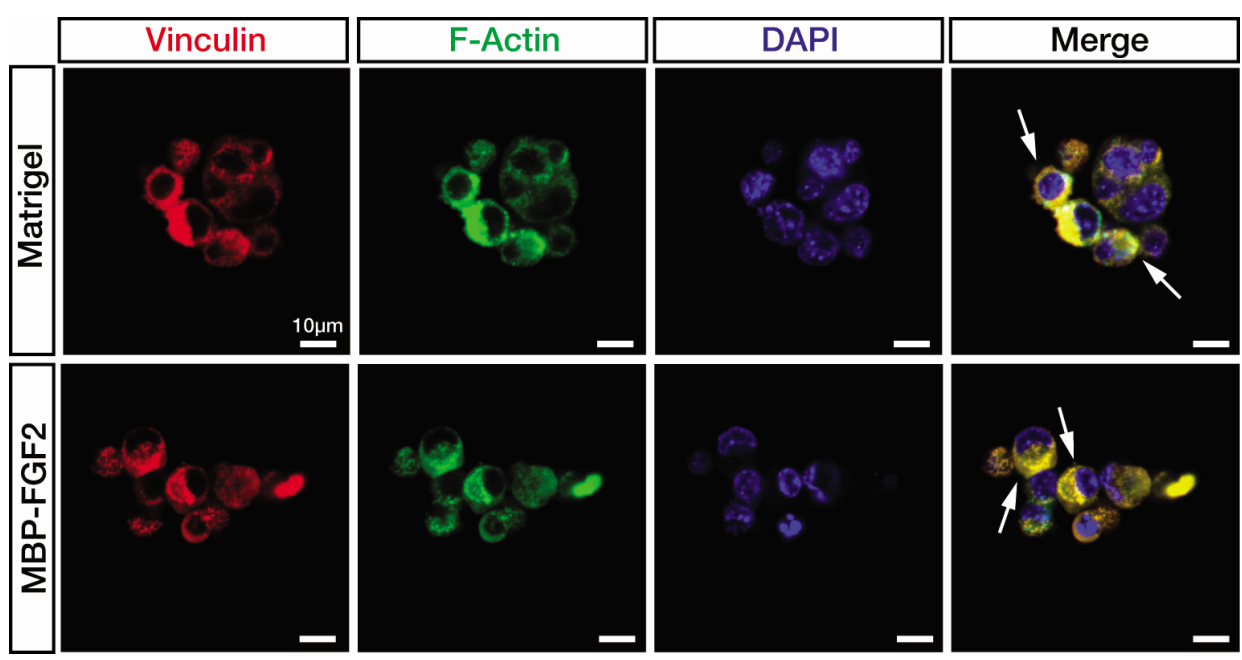

Fig. 4. Focal adhesion kinase expression is detected in the SkMSCs cultured on the MBP-FGF2-immobilized matrix. Confocal microscopic images of immunofluorescence staining of vinculin (red) and F-actin (green). SkMSCs were cultured on MBP-GF2 matrix (50 $\mu \mathrm{g} / \mathrm{mL})$ and Matrigel for $48 \mathrm{~h}$ in growth medium. White arrows indicate the cytoplasmic area overlapped between vinculin and F-actin expression. Scale bar, $10 \mu \mathrm{m}$.

entiation indices for the MBP-FGF2 matrix-precultured cells in a dose-dependent manner. Cell fusion indices showed no significant differences between Matrigel- and MBP-FGF2-precultured cells (Fig. 3C). Similar to differentiation indices, a minor increase in the fused myotube population was observed in the cells cultured on the matrix with high dose of MBP-FGF2. We do not know the nature of this mild effect, and further studies are warranted to determine whether SkMSCs precultured on the MBP-FGF2 matrix become more myogenic. Thus, the SkMSCs that expanded on the MBP-FGF2-immobilized matrix maintain robust myogenic potential equivalent to that of the cells precultured on Matrigel.

\section{Expression of focal adhesion (FA) kinase was comparable between SkMSCs cultured on Matrigel and MBP-FGF2 matrix}

Extracellular matrix is connected to the intracellular cytoskeleton through large integrin-based multi-protein complexes called FAs, which control cell morphology, migration, and adhesion (19). Vinculin is involved in anchoring F-actin to the cell membrane through its binding with ta- 
lin-integrin complex, leading to integrin clustering (20). To determine how FAs are distributed within the SkMSCs adhered to the immobilized MBP-FGF2, the expression of vinculin and F-actin was analyzed with immunofluorescence staining, followed by confocal microscopy. As shown in Fig. 4, vinculin expression was evenly distributed around the nucleus in the cells cultured on the MBP-FGF2-immobilized matrix and no significant difference in vinculin expression pattern was observed between these cells and those cultured on Matrigel. In both cases, vinculin expression was mostly co-localized with actin cytoskeleton. These results together with our previous findings $(12,13)$ suggest that HSPG on the surface of SkMSCs binds to the FGF2 moiety within the MBPFGF2-immobilized matrix.

In conclusion, the present study demonstrates that the MBP-FGF2-immobilized matrix may serve as a chemically well-defined and xeno-free cell culture substrate to support the adhesion, activation, and proliferation of SkMSCs and maintain their in vitro myogenic differentiation at the level comparable to that observed with the conventionally used Matrigel substrate. We failed to observe any significant dose-dependent difference with MBP-FGF2 concentrations ranging from 10 to $50 \mu \mathrm{g} / \mathrm{mL}$. We speculate that the immobilized MBP-FGF2 at $10 \mu \mathrm{g} / \mathrm{mL}$ of concentration is sufficient enough to support SkMSC behavior. The present study provides a proof-of-principle that a chemically well-defined, xeno-free, easy-to-use, and costeffective platform for the expansion of SkMSCs without the loss of their self-renewal and myogenic potential may be achieved with the MBP-FGF2-immobilized matrix. Currently, we are further improving the biofunctionality of this synthetic matrix by complexing with other chemically defined, xeno-free adhesion molecule(s) to improve initial cell attachment and proliferation of SkMSCs. Additionally, future engraftment experiments using SkMSCs expanded on the MBP-FGF2-immobilized matrix are warranted.

\section{Acknowledgments}

This research was supported by the Global Research Development Program grant (2016K1A4A3914725) and research grant (2016R1A2B4012956) from the National Research Foundation of Korea (NRF) to JK Yoon and the research grant (HI17C1193) from Korea Health Industry Development Institute (KHIDI) to Y Hwang.

\section{Potential Conflict of Interest}

The authors have no competing financial interest and potential conflicts of interest.

\section{References}

1. Morgan JE, Partridge TA. Muscle satellite cells. Int J Biochem Cell Biol 2003;35:1151-1156

2. Yin H, Price F, Rudnicki MA. Satellite cells and the muscle stem cell niche. Physiol Rev 2013;93:23-67

3. Hill M, Wernig A, Goldspink G. Muscle satellite (stem) cell activation during local tissue injury and repair. J Anat 2003;203:89-99

4. Seale P, Rudnicki MA. A new look at the origin, function, and "stem-cell" status of muscle satellite cells. Dev Biol 2000;218:115-124

5. Kuang S, Rudnicki MA. The emerging biology of satellite cells and their therapeutic potential. Trends Mol Med 2008;14:82-91

6. Charville GW, Cheung TH, Yoo B, Santos PJ, Lee GK, Shrager JB, Rando TA. Ex vivo expansion and in vivo self-renewal of human muscle stem cells. Stem Cell Reports 2015;5:621-632

7. Thomas K, Engler AJ, Meyer GA. Extracellular matrix regulation in the muscle satellite cell niche. Connect Tissue Res 2015;56:1-8

8. Bjornson CR, Cheung TH, Liu L, Tripathi PV, Steeper KM, Rando TA. Notch signaling is necessary to maintain quiescence in adult muscle stem cells. Stem Cells 2012; 30:232-242

9. Boldrin L, Neal A, Zammit PS, Muntoni F, Morgan JE. Donor satellite cell engraftment is significantly augmented when the host niche is preserved and endogenous satellite cells are incapacitated. Stem Cells 2012;30:1971-1984

10. Gilbert PM, Havenstrite KL, Magnusson KE, Sacco A, Leonardi NA, Kraft P, Nguyen NK, Thrun S, Lutolf MP, Blau HM. Substrate elasticity regulates skeletal muscle stem cell self-renewal in culture. Science 2010;329:10781081

11. Han WM, Anderson SE, Mohiuddin M, Barros D, Nakhai SA, Shin E, Amaral IF, Pêgo AP, García AJ, Jang YC. Synthetic matrix enhances transplanted satellite cell engraftment in dystrophic and aged skeletal muscle with comorbid trauma. Sci Adv 2018;4:eaar4008

12. Kang JM, Han M, Park IS, Jung Y, Kim SH, Kim SH. Adhesion and differentiation of adipose-derived stem cells on a substrate with immobilized fibroblast growth factor. Acta Biomater 2012;8:1759-1767

13. Kang J, Park HM, Kim YW, Kim YH, Varghese S, Seok HK, Kim YG, Kim SH. Control of mesenchymal stem cell phenotype and differentiation depending on cell adhesion mechanism. Eur Cell Mater 2014;28:387-403

14. Kim JH, Park Y, Jung Y, Kim SH, Kim SH. Combinatorial therapy with three-dimensionally cultured adipose-derived stromal cells and self-assembling peptides to enhance angiogenesis and preserve cardiac function in infarcted hearts. J Tissue Eng Regen Med 2017;11:2816-2827

15. Motohashi N, Asakura Y, Asakura A. Isolation, culture, and transplantation of muscle satellite cells. J Vis Exp 2014 Apr 8 [Epub]. doi: 10.3791/50846 
16. Michailovici I, Harrington HA, Azogui HH, YahalomRonen Y, Plotnikov A, Ching S, Stumpf MP, Klein OD, Seger R, Tzahor E. Nuclear to cytoplasmic shuttling of ERK promotes differentiation of muscle stem/progenitor cells. Development 2014;141:2611-2620

17. Han XH, Jin YR, Seto M, Yoon JK. A WNT/beta-catenin signaling activator, R-spondin, plays positive regulatory roles during skeletal myogenesis. J Biol Chem 2011;286: 10649-10659

18. Asfour HA, Allouh MZ, Said RS. Myogenic regulatory fac- tors: the orchestrators of myogenesis after 30 years of discovery. Exp Biol Med (Maywood) 2018;243:118-128

19. Humphries JD, Wang P, Streuli C, Geiger B, Humphries MJ, Ballestrem C. Vinculin controls focal adhesion formation by direct interactions with talin and actin. J Cell Biol 2007;179:1043-1057

20. Atherton P, Stutchbury B, Jethwa D, Ballestrem C. Mechanosensitive components of integrin adhesions: role of vinculin. Exp Cell Res 2016;343:21-27 